\title{
PROBLEMS ON ABELIAN FUNCTIONS AT THE TIME OF POINCARÉ AND SOME AT PRESENT ${ }^{1}$
}

\author{
BY JUN-ICHI IGUSA
}

This is an expanded version of our symposium lecture; it consists of two parts. In the first part we have tried to explain the problems on abelian functions at the time of Poincaré with a brief follow-up; in the second part we have explained, among others, a problem of Riemann and Weil on Jacobi's formula as one of the problems on abelian functions at present.

\section{Abelian functions by Poincaré.}

1-1. If the variable $x$ and a general solution $y$ of a linear differential equation with polynomial coefficients are algebraically dependent, the periods of abelian integrals of the first kind associated with the curve $f(x, y)=0$ satisfy certain relations. In his earliest works on abelian functions Poincaré examined such relations in some special cases. He also used a similar relation in a joint paper with Picard of 1883 on a "theorem of Riemann". Poincaré later developed a general theory of reducible integrals. This theory played some role in almost all of his works on abelian functions. We shall start by recalling the theorem of Riemann:

There are three related theorems concerning a complex torus. If $f$ is a meromorphic function on $\mathbf{C}^{g}$, an element $a$ of $\mathbf{C}^{g}$ such that $f(z+a)=f(z)$ for a variable $z$ in $\mathbf{C}^{g}$ is called a period of $f$; the set of all periods of $f$ forms a closed subgroup of $\mathbf{C}^{g}$, called the period group of $f$. Let $\Lambda$ denote a lattice in $\mathbf{C}^{\boldsymbol{g}}$, i.e., a discrete subgroup of $\mathbf{C}^{\mathbf{g}}$ with compact quotient; then a meromorphic function $f$ on $\mathbf{C}^{g}$ whose period group contains $\Lambda$, i.e., a meromorphic function on the complex torus $T=\mathbf{C}^{g} / \Lambda$ considered as a function on $\mathbf{C}^{g}$, is called an abelian function relative to $\Lambda$ and a holomorphic function $\Theta$ on $\mathbf{C}^{g}$ with the property

$$
\Theta(z+a)=\mathbf{e}\left(L_{a}(z)\right) \Theta(z)
$$

for every $a$ in $\Lambda$, in which $\mathbf{e}(t)$ stands for $\exp (2 \pi \sqrt{-1} t)$ and $L_{a}(z)$ is an affine linear function of $z$ depending on $a$, is called a theta function also relative to $\Lambda$.

Finally a complex $g$-by- $2 g$ matrix $\omega$ is called a Riemann matrix of degree $g$ if there exists a skew-symmetric integral matrix $C$ of degree $2 g$, called a principal matrix, such that

$$
\omega C^{t} \omega=0, \quad(1 / 2 \sqrt{-1}) \omega C^{t} \bar{\omega}>0
$$

Presented to the Symposium on the Mathematical Heritage of Henri Poincaré, April 7-10, 1980; received by the editors November $1,1980$.

1980 Mathematics Subject Classification. Primary 14K20; Secondary 14K25.

1 This work was partially supported by the National Science Foundation. 
the second condition means that the hermitian matrix $(1 / 2 \sqrt{-1}) \omega C^{t} \bar{\omega}$ is positive-definite. Such a matrix with

$$
C_{0}=\left(\begin{array}{cc}
0 & 1_{g} \\
-1_{g} & 0
\end{array}\right)
$$

as a principal matrix appeared in Riemann's paper [28] of 1857 as a period matrix of abelian integrals of the first kind associated with an algebraic curve of genus $g$. At any rate with this terminology the three theorems, called the Riemann-Weierstrass theorems, can be stated as follows:

(RW-1) Any g +1 abelian functions are algebraically dependent;

(RW-2) every abelian function $f$ can be expressed as a quotient of two theta functions;

(RW-3) if there exists an abelian function relative to $\Lambda$ with a discrete period group, any $g$-by- $2 g$ matrix $\omega$ whose $2 g$ columns generate $\Lambda$ is a Riemann matrix.

We recall that a divisor of a complex manifold is an integral linear combination of its subvarieties of codimension 1; it is called positive if the coefficients are nonnegative. The zeros and poles, counted with their multiplicities, of a meromorphic function $f \neq 0$ on the manifold are positive divisors; and their difference is called the divisor of $f$. The divisors of an abelian function $f \neq 0$ and a theta function $\Theta \neq 0$ may be considered as divisors of $T$. With this terminology $(\mathrm{RW}-2)$ can be replaced by the following theorem:

(RW-2 $2^{\#}$ Every positive divisor of $T$ is the divisor of a theta function $\Theta \neq 0$.

As a consequence, if $f \neq 0$ in (RW-2), the two theta functions can be chosen so that their divisors have no component in common. On the other hand the converse of (RW-3) is true and straightforward; in a paper of 1921 Lefschetz [16] proved the following theorem:

If $\omega$ is a Riemann matrix of degree $g$ and $\Lambda$ is the subgroup of $\mathbf{C}^{g}$ generated by its $2 g$ columns, necessarily a lattice in $\mathbf{C}^{g}$, there exists a finite set of theta functions giving rise to a biholomorphic map of $T=\mathbf{C}^{g} / \Lambda$ to a subvariety $A$ of a complex projective space.

And in 1949 Chow [5] proved the following theorem:

Every closed subvariety of a complex projective space is an algebraic variety and a meromorphic map between two such varieties is a rational map.

In particular the subvariety $A$ in the Lefschetz theorem is a smooth algebraic variety unique up to an isomorphism and it is called the abelian variety associated with $\omega$. If $\omega$ is a special Riemann matrix coming from an algebraic curve, the abelian variety $A$ is called the jacobian variety of the curve.

1-2. We shall now go back to 1883: it was known that Riemann had mentioned (RW-3) in a slightly different form to Hermite at the time of his visit to Paris in 1860. Hermite regarded the period relation in the general case 
as extremely remarkable and included it without proof in his note [10] to the sixth edition of Lacroix's text book, vol. 2, which appeared two years later. On the other hand, in 1869 Weierstrass published a short paper [36], in which (under the assumption in (RW-3)) he made the following statement: there exist $g+1$ algebraically dependent abelian functions by which every abelian function, all relative to $\Lambda$, can be rationally expressed. Actually he made a stronger statement and he later outlined a proof of the above statement in a letter to Borchardt of 1879 . We might mention that Weierstrass was not sure about (RW-2) in 1869 and became sure about it only in 1879; we might further mention that his detailed but not complete treatment [37] was published in 1903 after his death. Therefore although Riemann's paper of 1857 certainly was a classic in 1883 , the theory of general abelian functions was still up in the air at that time.

The short paper [20] by Poincaré and Picard was written under the above circumstances. In that paper they announced an outline of a proof of (RW-3), depending on (RW-1) by Weierstrass, and stated (RW-2) as its immediate consequence; there we see the fruitful idea to investigate $T$ via Riemann's theory applied to a suitable 1-dimensional subvariety of $T$. Later in 1891 Appell [2] gave a satisfactory proof of (RW-2) for $g=2$ by using Poincarés theorem of 1883 in the Acta Mathematica to the following effect: every meromorphic function on $\mathbf{C}^{2}$ can be expressed as a quotient of two holomorphic functions on $\mathbf{C}^{2}$. This theorem was generalized by Cousin in his thesis of 1895 and Appell's proof became valid for all $g$.

In 1897 Poincaré announced outlines of proofs of (RW-1) and (RW-2) in [24]; a detailed proof for (RW-2) appeared the following year in [25] and one for (RW-1) appeared five years later in [26]. The above second proof of (RW-2) by Poincaré was influenced by Appell; he simply modified the proof of his theorem in the Acta paper of 1883, on which Appell's proof depended, so that for an abelian function the two entire functions became theta functions. This proof was reproduced by Weil [40] in a Bourbaki seminar of 1949; Weil's proof was indeed a new proof in as much as the classical potential theory was eliminated. As for (RW-1) Poincare tried to show that the "image" of $T$ by $g+1$ abelian functions was algebraic; his idea to use the degrees of subvarieties of a complex projective space was interesting but the proof was not quite satisfactory. It was developed on a sound basis in the already mentioned paper by Chow of 1949. And in 1955 Siegel [35] gave a complete and yet elementary proof to the following general theorem:

Any $g+1$ meromorphic functions on a g-dimensional compact complex manifold are algebraically dependent; if there exist $g$ algebraically independent meromorphic functions, there exists another meromorphic function so that every meromorphic function on the manifold can be rationally expressed by those $g+1$ functions.

We must also mention Serre's influential works on this and other related theorems; as an example we refer to his Bourbaki talk [32] of 1954, which preceded his FAC and GAGA, for an elegant proof of Chow's theorem. 
1-3. We shall pass to reducible integrals: as we have recalled, Poincaré first worked on special examples; then he learned general theorems of Weierstrass through a paper by Kowalevski [14] of 1884. And he wrote [21] in the same year to supply proofs to those Weierstrass theorems and also to generalize them; two years later he wrote another paper [22] on reducible integrals with supplements and applications. In the following we shall review Poincaré's main theorem in its original form; but first we shall recall some definitions.

We say that two Riemann matrices $\omega_{1}, \omega_{2}$ of the same degree $g$ are equivalent if there exists a pair $(\lambda, L)$ of a complex invertible matrix $\lambda$ of degree $g$ and an integral matrix $L$ of degree $2 g$ satisfying $\lambda \omega_{1}=\omega_{2}^{t} L$. If we pass to the corresponding abelian varieties $A_{1}, A_{2}$, this means the existence of a surjective homomorphism from $A_{1}$ to $A_{2}$ necessarily with a finite kernel. At any rate we have an equivalence relation in the set of all Riemann matrices of degree $g$.

We recall that every nonsingular skew-symmetric integral matrix $C$ of degree $2 g$ can be written as $C={ }^{t} L C_{0} L$ for some integral matrix $L$ of degree $2 g$; in particular if we define the $\operatorname{Pfaffian} \operatorname{Pf}(C)$ of $C$ as $|\operatorname{det}(L)|$, we get $\operatorname{det}(C)=\operatorname{Pf}(C)^{2}$. If $\omega$ is a Riemann matrix with $C$ as a principal matrix, necessarily $C$ is nonsingular and in the above notation $\omega$ is equivalent to $\omega^{t} L$; the point is that $\omega^{t} L$ has $C_{0}$ as a principal matrix. Therefore every equivalence class of Riemann matrices contains one with $C_{0}$ as a principal matrix.

If a Riemann matrix $\omega$ has $C_{0}$ as a principal matrix, its right square submatrix $\lambda$ is invertible and if we write $\omega$ as $\omega=\lambda\left(\tau 1_{g}\right)$, we will have

$$
{ }^{t} \tau=\tau, \quad \operatorname{Im}(\tau)>0
$$

the second condition means that the imaginary part of $\tau$ is positive-definite. The set $\mathbf{S}_{g}$ of all such $\tau$ 's forms an open convex cone in the vector space of symmetric complex matrices of degree $g$; and in honor of his fundamental works, especially $[33,34]$, it is called the Siegel upper-half space of degree $g$. If $M$ is a real matrix of degree $2 g$ satisfying ${ }^{t} M C_{0} M=C_{0}$ and if we write

$$
M=\left(\begin{array}{ll}
a & b \\
c & d
\end{array}\right)
$$

with submatrices $a, b, c, d$ of degree $g$, then

$$
M \cdot \tau=(a \tau+b)(c \tau+d)^{-1}
$$

defines a holomorphic action of the real symplectic group $S p_{2 g}(\mathbf{R})$ on $\mathbf{S}_{g}$. Poincaré's formulation of his "complete reducibility theorem" involves the action of $S p_{2 g}(\mathbf{Q})$ on $\mathbf{S}_{g}$ and it is as follows:

(P-1) Let $\omega$ denote a Riemann matrix of degree $g$ with $C_{0}$ as a principal matrix such that for some complex $g^{\prime}$-by- $2 g^{\prime}$ matrix $\omega^{\prime}$ and for some integral $2 g$-by- $2 g^{\prime}$ matrix $R$, where $1 \leqslant g^{\prime}<g$, the upper $g^{\prime}$-by- $2 g$ submatrix of $\omega$ can be written as $\omega^{\prime}{ }^{t} R$; then there exist an element $M$ of $S p_{2 g}(\mathbf{Q})$ and a point $\tau^{\prime}$ of $\mathbf{S}_{g^{\prime}}$ such that $\left(\tau^{\prime} 1_{g^{\prime}}\right)$ is equivalent to the necessarily Riemann matrix $\omega^{\prime}$ and

$$
M \cdot \tau=\left(\begin{array}{cc}
\tau^{\prime} & 0 \\
0 & \tau^{\prime \prime}
\end{array}\right)
$$

in which $\omega=\lambda\left(\tau 1_{g}\right)$. 
It follows from (P-1) that the equivalence class of an arbitrary Riemann matrix contains one of the form

$$
\left(\begin{array}{llll}
\omega^{\prime} & & & \\
& \omega^{\prime \prime} & & \\
& & \ddots & \\
& & & \omega^{(r)}
\end{array}\right),
$$

in which $\omega^{\prime}, \omega^{\prime \prime}, \ldots, \omega^{(r)}$ are irreducible or pure Riemann matrices. However Poincaré was not aware of the uniqueness of the classes of those component matrices; this was later observed by Scorza; cf. Albert [1].

An outline of Poincarés proof of (P-1) in the current terminology is as follows: we regard elements of $\mathbf{Q}^{2 g}$ as column vectors and convert $\mathbf{Q}^{2 g}$ into a symplectic space over $Q$ via the skew-symmetric bilinear form $B(x, y)$ $={ }^{t} x C_{0} y$; then the columns of $R$ span a $2 g^{\prime}$-dimensional nondegenerate, hence symplectic, subspace. Therefore we can find a symplectic basis $x_{1}, \ldots, x_{g}$, $y_{1}, \ldots, y_{g}$ for $\mathbf{Q}^{2 g}$ such that $x_{1}, \ldots, x_{g^{\prime}}, y_{1}, \ldots, y_{g^{\prime}}$ form a symplectic basis for that subspace. If we define $M$ as the inverse of the square matrix of degree $2 g$ with $x_{1}, \ldots, x_{g}, y_{1}, \ldots, y_{g}$ as its columns, then $M$ has the required property.

Actually Poincaré considered $\mathbf{Z}^{2 g}$ as a symplectic module over $\mathbf{Z}$ via the same $B(x, y)$ and tried to find a suitable $\mathbf{Z}$-basis for the submodule generated by the columns of $R$; and, e.g., in the simplest case where $g^{\prime}=1$ he showed the existence of $M$ in $S p_{2 g}(Z)$ such that the first row of $M \cdot \tau$ took the form

$$
\left(\tau^{\prime} k^{-1} \mu 0 \ldots 0\right)
$$

in which $k=\operatorname{Pf}\left({ }^{t} R C_{0} R\right)$ and $0 \leqslant \mu<k$. This is equivalent to a theorem of Weierstrass; cf. [14, p. 400].

At any rate, as an immediate consequence of (P-I), Poincaré observed that the $S p_{2 g}(\mathbf{Q})$-orbit of $\sqrt{-1} 1_{g}$ was dense in $\mathbf{S}_{g}$; cf. [22, p. 340]. He regarded this fact as a key to solve various problems: "C'est là une circonstance qui donnera, je n'en doute pas, la clef de bien des problèmes".

1-4. We shall explain how Poincaré applied the above observation to a problem on theta functions; we shall first recall a theorem of Frobenius: let $\Theta$ denote a theta function relative to a lattice $\Lambda$ in $\mathbf{C}^{g}$ and denote the linear part $L_{a}(z)-L_{a}(0)$ of $L_{a}(z)$ by $\lambda_{a}(z)$; then the difference $B(a, b)=\lambda_{a}(b)-\lambda_{b}(a)$ is an integer for every $a, b$ in $\Lambda$. Therefore if we choose a $\mathbf{Z}$-basis $a_{1}, \ldots, a_{2 g}$ for $\Lambda$, we get a skew-symmetric integral matrix $E$ of degree $2 g$ with $B\left(a_{i}, a_{j}\right)$ as its $(i, j)$ th coefficient. And if $E$ is nonsingular, then $\omega=\left(a_{1} \ldots a_{2 g}\right)$ becomes a Riemann matrix with $E^{-1}$, multiplied by a suitable positive integer, as a principal matrix. Furthermore the set of all theta functions relative to $\Lambda$ with the same $\mathbf{e}\left(L_{a}(z)\right)$ forms a vector space over $\mathbf{C}$ and, according to Frobenius [8], its dimension is equal to $\operatorname{Pf}(E)$.

Poincare consistently restricted his attention to the case where $E=-k C_{0}$ for some positive integer $k$; in that case the dimension is $k^{g}$. A theta function with $E=-k C_{0}$ is called a theta function of order $k$; for such theta functions we may assume that $\Lambda$ is the lattice generated by the $2 g$ columns of $\left(\tau 1_{g}\right)$, in 
which $\tau$ is in $\mathbf{S}_{g}$. In a paper [19] of 1883 and later in [22] already mentioned, Poincaré proved the following theorems:

(P-2) Let $\Theta_{1}, \ldots, \Theta_{g}$ denote $g$ theta functions of orders $k_{1}, \ldots, k_{g}$; then the number $N$ of common zeros, each counted with its multiplicity, of

$$
\Theta_{1}\left(z-b_{1}\right), \ldots, \Theta_{g}\left(z-b_{g}\right)
$$

in $T=\mathbf{C}^{g} / \Lambda$ is equal to $k_{1} \ldots k_{g} g$ ! provided that it is finite;

(P-3) let $\zeta_{1}, \ldots, \zeta_{N}$ denote representatives in $\mathbf{C}^{g}$ of the common zeros; then

$$
\sum_{i=1}^{N} \zeta_{i} \equiv k_{1} \ldots k_{g}(g-1) ! \sum_{j=1}^{g} b_{j}+c \bmod \Lambda,
$$

in which $c$ is independent of $b_{1}, \ldots, b_{g}$.

The above theorems were later generalized by Wirtinger [41]. Although they are now well understood, Poincaré's proofs are still interesting: he observed in (P-2) that the number $N$ was a continuous function of $\tau$ and the parameters in $L_{a}(z)$; hence $N$ was a constant. In order to determine this constant he specialized $\tau$ to a diagonal matrix and thus reduced the general case to the elliptic case where $g=1$.

The proof of (P-3) is more involved: by using a generalization of Abel's theorem he showed that the sum depended continuously and only on $\tau$ and the parameters in $L_{a}(z)$ once $b_{1}, \ldots, b_{g}$ were fixed. By using the transformation theory he then showed that the formula to be proved was invariant under the action of $S p_{2 g}(\mathbf{Q})$. On the other hand the $S p_{2 g}(\mathbf{Q})$-orbit of $\sqrt{-1} 1_{g}$ was already shown to be dense in $\mathbf{S}_{g}$. Therefore it was enough to verify the formula again in the case where $\tau$ was a diagonal matrix.

1-5. We have reviewed Poincaré's idea to use $\left(\mathbf{S}_{1}\right)^{g}=\mathbf{S}_{1} \times \cdots \times \mathbf{S}_{1}$ embedded in $\mathbf{S}_{g}$; he also used an infinitesimal neighborhood of $\left(\mathbf{S}_{1}\right)^{g}$ in his paper [23] of 1895. In that paper he examined the condition for $\tau$ to be a "jacobian point", i.e., a point of $\mathbf{S}_{g}$ coming from an algebraic curve of genus $g$. This problem was treated by Schottky [31] seven years earlier in the first nontrivial case where $g=4$. Poincaré's method is entirely different from Schottky's and it is as follows:

First of all any theta function of order 1 can be converted into

$$
\theta(z)=\sum_{p \in \mathbf{Z}^{8}} \mathbf{e}\left(\left(\frac{1}{2}\right)^{t} p \tau p+{ }^{t} p z\right)
$$

the conversion consists of an affine linear transformation in $\mathbf{C}^{g}$ and of a multiplication by a "trivial theta functions", i.e., the exponential of a polynomial of degree 2 in the coefficients of $z$. We shall denote by $\tau_{i j}$ and $z_{i}$ the $(i, j)$ th coefficient and the $i$ th coefficient of $\tau$ and $z$, respectively. If the off-diagonal or lateral coefficients $\tau_{i j}, i \neq j$, of $\tau$ are all zero, we will have

$$
\theta(z)=\theta_{1}\left(z_{1}\right) \ldots \theta_{g}\left(z_{g}\right)
$$


in which

$$
\theta_{i}(u)=\sum_{p=-\infty}^{\infty} \mathbf{e}\left(\left(\frac{1}{2}\right) \tau_{i i} p^{2}+p u\right)
$$

for $1 \leqslant i \leqslant g$.

A hypersurface in $\mathbf{C}^{g}$ is called a translation surface if at every point of the hypersurface its neighborhood admits a parametric representation of the form

$$
z=\sum_{i=1}^{g-1} \phi_{i}\left(t_{i}\right)
$$

in which $\phi_{1}, \ldots, \phi_{g-1}$ are holomorphic functions of one variable. If $\tau$ is a jacobian point, the hypersurface in $\mathbf{C}^{g}$ defined by $\theta(z)=0$ is a translation surface; this follows from a theorem of Riemann. Poincaré took a point $\tau$ of $\mathbf{S}_{\boldsymbol{g}}$ with very small lateral coefficients and examined the condition that $\theta(z)=0$ defined a translation surface in a very small neighborhood of $\zeta=\left(\zeta_{1}, \ldots, \zeta_{g}\right)$ where $\theta_{i}\left(\zeta_{i}\right)=0$ for $1 \leqslant i \leqslant g$. And in the case where $g=4$ he proved the following theorem:

(P-4) Put $a_{i j}=2 \pi \sqrt{-1} \tau_{i j}, \quad r_{1}=a_{12} a_{13} a_{42} a_{43}, \quad r_{2}=a_{13} a_{14} a_{23} a_{24}, \quad r_{3}=$ $a_{14} a_{12} a_{34} a_{32}$, and

$$
R=r_{1}^{2}+r_{2}^{2}+r_{3}^{2}-2\left(r_{2} r_{3}+r_{3} r_{1}+r_{1} r_{2}\right)
$$

then $R(\tau)$ becomes a higher order infinitesimal if $\tau$ is a jacobian point with very small lateral coefficients.

Actually Poincaré wrote down his condition in an irrational form, but the two conditions are equivalent. Schottky, on the other hand, found a modular form $J$ of weight 8 for the full modular group $S p_{8}(\mathbf{Z})$ such that $J(\tau)=0$ at every jacobian point $\tau$. If we expand $J$ at a variable point $\tau$ in which the lateral coefficients are very small, we get

$$
J=2^{-16} \cdot \prod_{i=1}^{4}\left(\theta_{00} \theta_{01} \theta_{10}\right)_{i}(0)^{8} \cdot R+\cdots ;
$$

the $\theta_{i j}(u)$ for $i, j=0,1$ are the standard elliptic theta functions and the subscript $i$ on the right-hand side indicates that the modulus is $\tau_{i i}$. The relation of the conditions found by Poincare and Schottky was discussed by Rauch [27] with some comments about their proofs.

1-6. Poincaré's works on abelian functions proper ended by his paper of 1902. However a consequence of his later papers of 1910-1911 on normal functions has to be mentioned. As we have recalled, if $V$ is an algebraic curve of genus $g$, the complex torus associated with its period matrix is biholomorphic to an abelian variety $J$, called the jacobian variety of $V$. It follows from Abel's theorem that $J$ can be obtained also as follows: we take the group of divisors of $V$ each with the property that the sum of its coefficients is 0 . This group contains the group of divisors of rational functions on $V$, different from the constant 0 , and the quotient group with a natural structure of an algebraic variety becomes an abelian variety isomorphic to $J$. 
If we start from an algebraic surface as in Poincaré or more generally from any smooth algebraic variety $V$, then the period matrix of simple integrals of the first kind on $V$ can be obtained by reducing the period matrix associated with a general curve on $V$ and, in particular, it is a Riemann matrix; thus we get an abelian variety $A$ which generalizes $J$. Another aspect of $J$ can also be generalized. We take the group of divisors of $V$ each homologous to 0 as an integral cycle. This group contains the group of divisors of rational functions on $V$, different from the constant 0 , and the quotient group again becomes an abelian variety, say $P$. Poincaré's theory of normal functions implies the existence of a surjective homomorphism from $A$ to $P$ with a finite kernel, i.e., the Riemann matrices of $A$ and $P$ are equivalent. This was really an important achievement in the theory of algebraic varieties at that time. In the general case, however, the abelian varieties $A$ and $P$, called the Albanese and the Picard varieties of $V$, respectively, are not isomorphic; and the exact relation between $A$ and $P$ had been left in the shadows until our thesis [11] of 1952.

We shall conclude the first part with the following comments: first of all in his paper of 1938 Weil [38] introduced "hyperabelian functions"; this theory has not yet been fully explored. On the other hand if we pass to abelian varieties, the theory of abelian functions loses its transcendental character. And in fact a purely algebraic theory of abelian functions over an algebraically closed field of arbitrary characteristic was created by Weil [39] in 1948. Also a purely algebraic theory of theta functions was developed during 1966 - 1967 by Mumford [17]. We might mention that their works contain generalizations and new results even in the complex case. Finally we mention that Mumford's lecture note [18] and Freitag's report [7] will give a good coverage of the results and problems up until 1976.

\section{A problem of Riemann and Weil.}

2-1. We shall start by recalling some definitions: let $\Theta$ denote a theta function of order 1; then, after a linear transformation in $\mathbf{C}^{g}$ and a multiplication by a trivial theta function, it becomes

$\theta_{m}(\tau, z)=\sum_{p \in \mathbf{Z}^{8}} \mathbf{e}\left(\left(\frac{1}{2}\right)^{t}\left(p+\frac{1}{2} m^{\prime}\right) \tau\left(p+\frac{1}{2} m^{\prime}\right)+{ }^{t}\left(p+\frac{1}{2} m^{\prime}\right)\left(z+\frac{1}{2} m^{\prime \prime}\right)\right)$,

in which $m$ is the column vector with $m^{\prime}, m^{\prime \prime}$ in $\mathbf{R}^{g}$ as its first and the second entry vectors. If $m=0$, we get back to the theta function $\theta(z)$ in 1-5. We shall assume that $m$ has integral coefficients; then we have

$$
\theta_{m}(\tau,-z)=e(m) \theta_{m}(\tau, z), \quad e(m)=(-1)^{t_{m^{\prime} m^{*}}}
$$

Therefore $\theta_{m}(\tau, z)$ is even or odd according as the "characteristic" $m$ is even or odd in the sense that $e(m)= \pm 1$. In view of $\theta_{m+2 n}(\tau, z)= \pm \theta_{m}(\tau, z)$ for every $n$ in $\mathbf{Z}^{2 g}$ we may assume, if necessary, that the coefficients of $m$ are 0,1 . The number of theta functions then becomes $2^{2 g}$; of these $2^{g-1}\left(2^{g}+1\right)$ are even and the remaining $2^{g-1}\left(2^{g}-1\right)$ are odd. If $m$ is even, we put $\theta_{m}(\tau)=$ $\theta_{m}(\tau, 0)$; the holomorphic function $\theta_{m}$ on $\mathbf{S}_{g}$ so defined is called a Thetanullwert. We shall denote by $\mathbf{C}[\theta]$ the ring generated over $\mathbf{C}$ by the Thetanullwerte. 
We shall change our notation in Part 1 and denote an element of $S p_{2 g}(\mathbf{R})$ by $\sigma$ instead of $M$; however we shall denote its submatrices by $a, b, c, d$ as before. If $l$ is a positive integer, we define a normal subgroup $\Gamma_{g}(l)$ of $S p_{2 g}(Z)$ by the condition $\sigma \equiv 1_{2 g} \bmod l$. We further define a subgroup $\Gamma_{g}(l, 2 l)$ of $\Gamma_{g}(l)$ by the additional condition

$$
\operatorname{diag}\left(a^{t} b\right) \equiv \operatorname{diag}\left(c^{t} d\right) \equiv 0 \bmod 2 l
$$

we have denoted by "diag" the conversion of a square matrix into a column vector by its diagonal coefficients. If $l$ is even, then $\Gamma_{g}(l, 2 l)$ is normal in $\Gamma_{g}(1)=S p_{2 g}(\mathbf{Z})$.

The correspondence $(\sigma, \tau) \rightarrow \operatorname{det}(c \tau+d)$ defines a holomorphic automorphy factor of $S p_{2 g}(R)$; and if $\sigma$ is in $\Gamma_{g}(4)$, then

$$
\operatorname{det}(c \tau+d)^{1 / 2}=\theta(\sigma \cdot \tau) \theta(\tau)^{-1}
$$

defines a holomorphic automorphy factor of $\Gamma_{g}(4)$. If we have a power $\rho(\sigma, \tau)$ of such an automorphy factor of a subgroup $\Gamma$ of $S p_{2 g}(\mathbf{R})$, we can let $\Gamma$ act on the vector space of all holomorphic functions on $\mathbf{S}_{g}$ as

$$
(\sigma \cdot f)(\tau)=\rho\left(\sigma^{-1}, \tau\right)^{-1} f\left(\sigma^{-1} \cdot \tau\right) .
$$

If $\Gamma$ is a modular group, i.e., a subgroup of $\Gamma_{g}(1)$ containing $\Gamma_{g}(l)$ for some $l$, any $\Gamma$-invariant function, which satisfies a regularity condition at "cusps" in the case $g=1$, is called a modular form relative to $\Gamma$. If $\rho(\sigma, \tau)$ is the $k$ th power of $\operatorname{det}(c \tau+d)$ for some nonnegative integer $k$, we say that the modular form $f$ has weight $k$; and if $\rho(\sigma, \tau)$ is the $k$ th power of $\operatorname{det}(c \tau+d)^{1 / 2}$ for some $\Gamma$ contained in $\Gamma_{g}(4)$, we say that $f$ has degree $k$. For instance every Thetanullwert is a modular form of degree 1 relative to $\Gamma_{g}(4,8)$. We shall denote by $A(\Gamma)$ the ring consisting of finite sums of modular forms relative to $\Gamma$; then $A(\Gamma)$ forms a graded integral domain over $\mathbf{C}$ and we have the following theorem:

The graded ring $A\left(\Gamma_{8}(4,8)\right)$ is precisely the integral closure of $\mathrm{C}[\theta]$ within its field of fractions.

This theorem was proved in [12] by using the theory of compactifications by Baily [3] and Cartan [4]. The two rings in the theorem are the same for $g \leqslant 2$ but are different for $g \geqslant 3$; and yet the morphism

$$
\operatorname{proj}\left(A\left(\Gamma_{g}(4,8)\right)\right) \rightarrow \operatorname{proj}(\mathbf{C}[\theta])
$$

- the notation will be explained in a moment-is always bijective.

2-2. We shall recall the main theorem in the theory of compactifications: if $A=A_{0}+A_{1}+A_{2}+\cdots$ is a finitely generated graded integral domain over $A_{0}=\mathrm{C}$, the set of homogeneous maximal ideals of $A$ other than $A_{1}+A_{2}+\cdots$ becomes a projective algebraic variety $\operatorname{proj}(A)$. The graded ring $A(\Gamma)$ for any modular group $\Gamma$ of degree $g$ is such a ring and it is integrally closed. Therefore $\operatorname{proj}(A(\Gamma))$ is defined and it is a normal variety. Furthermore the quotient variety $\Gamma \backslash \mathbf{S}_{\mathrm{g}}$ is biholomorphic to a Zariski open subset of $\operatorname{proj}(A(\Gamma))$ such that the complement is a finite union of $\operatorname{proj}\left(A\left(\Gamma^{\prime}\right)\right)$ for some modular groups $\Gamma^{\prime}$ of degrees less than $g$. Since $\operatorname{proj}(A(\Gamma))$ for $\Gamma=\Gamma_{g}(1)$ was first introduced by Satake [30] as a Hausdorff space, it is 
sometimes called the Satake compactification of $\Gamma \backslash \mathbf{S}_{g}$. At any rate the main theorem and other standardized theorems imply that for $g \geqslant 2$ every meromorphic function on $\Gamma \backslash \mathbf{S}_{g}$ can be expressed as a quotient of two modular forms and in particular every holomorphic function on $\Gamma \backslash \mathbf{S}_{g}$ is a constant.

We shall explain three problems; the first one is called the Schottky problem:

Find an explicit description of a finite basis for the homogeneous ideal of $A\left(\Gamma_{g}(1)\right)$ generated by those modular forms which vanish at every jacobian point.

Since the ideal is $\{0\}$ for $g \leqslant 3$, the first nontrivial case is $g=4$; even in that case the problem is not quite settled because no proof has yet been published for the fact that the Schottky invariant $J$ generates the ideal. The second problem is related to Poincaré [21, 22] and also to Kowalevski [14].

Find an explicit description of a finite basis for the homogeneous ideal of $A\left(\Gamma_{g}(1)\right)$ generated by those modular forms which vanish at every reducible point.

The second problem seems much easier than the first problem; and yet answers have been given only for $g \leqslant 3$. The third problem is a refinement of the main theorem and it is as follows:

Denote by $A_{\mathrm{z}}\left(\Gamma_{g}(1)\right)$ the ring consisting of finite sums of modular forms relative to $\Gamma_{g}(1)$ with integral Fourier coefficients: show that $A_{\mathbf{z}}\left(\Gamma_{g}(1)\right)$ is finitely generated over $\mathbf{Z}$.

This problem has to be completed as follows: show also that $\operatorname{proj}\left(A_{\mathbf{Z}}\left(\Gamma_{g}(1)\right)\right)$ gives the "scheme of moduli" of all "principally polarized abelian varieties" of dimensions at most equal to $g$. If the problem is settled in the so-completed form, then that will imply the irreducibility of the variety of moduli over an algebraically closed field of arbitrary characteristic. At any rate the third problem has been settled only for $g \leqslant 2$; in those two cases the structure of $A_{\mathbf{z}}\left(\Gamma_{g}(1)\right)$ is also known.

2-3. We shall now explain a problem of Riemann and Weil; first we shall introduce a notation. Let $m_{1}, \ldots, m_{g}$ denote $g$ odd characteristics and put

$$
D(M)(\tau)=\pi^{-g}\left(\partial\left(\theta_{m_{1}}, \ldots, \theta_{m_{g}}\right) / \partial\left(z_{1}, \ldots, z_{g}\right)\right)(\tau, 0),
$$

in which $M$ stands for the $2 g$-by-g matrix $\left(m_{1} \ldots m_{g}\right)$; this is the Nullwert of the jacobian of $\theta_{m_{1}}(\tau, z), \ldots, \theta_{m_{8}}(\tau, z)$ with the normalizing factor $\pi^{-g}$ and it is a holomorphic function on $\mathbf{S}_{g}$. In a letter dated April 14, 1976 Weil proposed the following problem:

(W-1) Is $D(M)$ always a polynomial in the Thetanullwerte with integral rational coefficients?

At that time he needed an affirmative answer to the following weaker problem:

(W-2) Can $D(M)$ be expressed as a quotient of two such polynomials?

In the same letter he referred to Frobenius [9] and a quotation by M. Noether from Riemann's manuscripts [29, p. 66] for the background of his 
problems. Noether found in "Nr. 25, Bogen 6" expressions for $D(M)$ as "Summen von Produkten" of $g+2$ Thetanullwerte for $g=3,4, \ldots, 7$; this was quoted later in Krazer and Wirtinger [15, p. 772]. Frobenius, on the other hand, gave a survey of earlier results, unaware of Riemann's manuscripts, and proved such formulas for $g \leqslant 4$ with a final detail for $g=4$ left as a conjecture; this was nineteen years after Riemann's death. Actually for $g=3$, 4 Frobenius proved the formulas under certain conditions on $m_{1}, \ldots, m_{g}$.

Our first result on the above problems was that (W-2) had an affirmative answer; an outline of the proof is as follows: if we denote by $\mathbf{Q}[\theta]$ the ring generated over $\mathbf{Q}$ by the Thetanullwerte, then $\mathbf{Q}[\boldsymbol{\theta}]$ is a $\mathbf{Q}$-structure on $\mathbf{C}[\boldsymbol{\theta}]$. Let $\mathbf{Q}[\theta]^{*}$ denote the integral closure of $\mathbf{Q}[\theta]$; then a theorem in algebraic geometry tells us that the $\mathbf{C}$-span of $\mathbf{Q}[\theta]^{*}$ is the integral closure of $\mathbf{C}[\theta]$, which by our theorem in 2-1 coincides with $A\left(\Gamma_{g}(4,8)\right.$ ); and $D(M)$ is a homogeneous element of $A\left(\Gamma_{g}(4,8)\right.$ ) of degree $g+2$. Finally $D(M)$ is in $\mathbf{Q}[\theta]^{*}$ because its Fourier coefficients are all integers.

2-4. We shall next explain our theorem in [13]; we shall use the following terminology and notation: a sequence of $2 g+2$ characteristics $m_{1}, \ldots, m_{g}, n_{1}, \ldots, n_{g+2}$ is called a special fundamental system if $m_{1}, \ldots, m_{g}$ are odd, $n_{1}, \ldots, n_{g+2}$ are even, and for every $a, b, c$ in the sequence

$$
e(a) e(b) e(c) e(a+b+c)=-1
$$

holds. We put $M=\left(m_{1} \ldots m_{g}\right)$ as before and $N=\left(n_{1} \ldots n_{g+2}\right)$; we also put

$$
P(N)=\theta_{n_{1}} \ldots \theta_{n_{z+2}} .
$$

Finally for any integral domain $R$ and any positive integer $r$ we shall denote by $O_{r}(R)$ the orthogonal group of $x_{1}^{2}+\cdots+x_{r}^{2}$ over $R$ and by $G_{r}, \Pi_{r}$ the subgroups of $\mathbf{O}_{r}\left(\mathbf{F}_{2}\right)$ obtained from $\mathbf{O}_{r}\left(\mathbf{Z}_{2}\right), \mathbf{O}_{r}(\mathbf{Z})$, respectively, under the reduction mod 2; we have denoted by $Z_{2}$ the ring of 2-adic integers and by $F_{2}$ the field with two elements. Then our theorem can be stated as follows:

(J-1) If $D(M)$ is different from the constant 0 and is a polynomial in the Thetanullwerte, then for some $N$ the $2 g+2$ columns of $(M N)$ form a special fundamental system and the formula

$$
D(M)=\sum_{V \in G_{g+2} / \Pi_{g+2}} \pm P(N V)
$$

holds; the expression on the right-hand side is unique.

Since the formula is unique, all known formulas have to be that formula; and by Riemann it has to be correct up to $g=7$. We tried, therefore, to remove the ugly "if" in our theorem; but we were able to prove the formula only up to $g=5$ by the method of Frobenius with a consequence of the theory of compactifications to give a finishing touch.

In a paper of 1979, which we completely overlooked, Fay [6] also proved the formula in $(\mathrm{J}-1)$ up to $g=5$. Furthermore by using some properties of theta functions with a hyperelliptic modulus he discovered the following remarkable fact:

(F) The formula in (J-1) does not hold for $g=6$. 
Fay's finding was a relative impossibility. However if we put (J-1) and (F) together, we get the following absolute impossibility: for $g=6$ no $D(M)$ other than the constant 0 is a polynomial in the Thetanullwerte. Therefore (W-1) has a negative answer and the quotations of Riemann by Noether and by Krazer and Wirtinger are all wrong! We have tried to remedy this unfortunate situation and found a right formulation of (J-1); and it is as follows:

$\left(\mathrm{J}-1^{\#}\right)$ Let $X$ and $Y$ denote the C-spans of all $D(M)$ 's and $P(N)$ 's, respectively; then the dimension of $X \cap Y$ is either 0 or equal to $\operatorname{card}\left(S p_{2 g}\left(\mathbf{F}_{2}\right)\right) / \operatorname{card}\left(G_{g} \times G_{g+2}\right)$. Furthermore in the second case it has a C-basis consisting of elements of the form

$$
\sum_{U \in G_{g} / \Pi_{g}} \pm D(M U)=\sum_{V \in G_{g+2} / \Pi_{g+2}} \pm P(N V)
$$

in which the expressions on both sides are unique up to a simultaneous sign change.

This theorem can be proved in the same way as (J-1); cf. [13, pp. 440-441]. The only difference is that we incorporate the results in the Appendix of that paper where we observed the "symmetry" in $M$ and $N$ of such formulas. At any rate $\left(\mathrm{J}-1^{\sharp}\right)$ implies $(\mathrm{F})$ : if $g=6$ or more generally if $g \geqslant 6$, then $\left[G_{g}: \Pi_{g}\right] \geqslant 2$, hence by the uniqueness no $D(M)$ can be written as $\Sigma \pm$ $P(N V)$.

2-5. By what we have explained we have $\operatorname{dim}(X \cap Y) \geqslant 1$ for $g \leqslant 5$ while $\operatorname{dim}(X \cap Y)$ is either 0 or

$$
2^{18} 3^{4} 5 \cdot 7 \cdot 11 \cdot 13 \cdot 17 \cdot 31=56,006,655,344,640
$$

for $g=6$; our conjecture is that $\operatorname{dim}(X \cap Y) \geqslant 1$ for all $g$. We have examined this conjecture from a representation-theoretic viewpoint by using Mackey's theorem. We can easily see that if the conjecture is true for a certain $g \geqslant 2$, it is true also for $g-1$. Therefore we may assume that $g$ is even; then we can let $\Gamma_{g}(1)$ act on $X$ and $Y$ via the $\left(\frac{1}{2} g+1\right)$ th power of $\operatorname{det}(c \tau+d)$ as an automorphy factor. We see that all $D(M)$ 's and $P(N)$ 's are eigenvectors for the representations of $\Gamma_{g}(2)$ in $X$ and $Y$, hence they give rise to its characters, say $\psi_{M}$ and $\psi_{N}$. The point is that we have $\psi_{M}=\psi_{N}$ if and only if the $2 g+2$ columns of $(M N)$ form a special fundamental system. Furthermore if we denote such a character by $\psi$, all solutions of $\psi_{M^{\prime}}=\psi$ can be written as $M^{\prime} \equiv M U \bmod 2$ with $U \bmod 2$ in $G_{g}$; similarly all solutions of $\psi_{N^{\prime}}=\psi$ can be written as $N^{\prime} \equiv N V \bmod 2$ with $V \bmod 2$ in $G_{g+2}$. Finally if we denote by $\Gamma$ the subgroup of $\Gamma_{g}(1)$ consisting of all $\sigma$ in $\Gamma_{g}(1)$ satisfying

$$
\psi\left(\sigma \sigma_{0} \sigma^{-1}\right)=\psi\left(\sigma_{0}\right)
$$

for every $\sigma_{0}$ in $\Gamma_{g}(2)$, then $\Gamma / \Gamma_{g}(2)$ is isomorphic to $G_{g} \times G_{g+2}$; and we get the following theorem:

(J-2) There exists a degree 1 character $\chi$ of $\Gamma$ which extends $\psi$; the representations of $\Gamma_{g}(1)$ in $X$ and $Y$ share one and only one irreducible representation $\rho$ with multiplicity one and $\rho$ is the representation of $\Gamma_{g}(1)$ induced 
by $\chi$. Furthermore the $\left[\Gamma_{g}(1): \Gamma\right]$ elements which appear on the left- and right-hand sides of the formula in $\left(\mathrm{J}-1^{\sharp}\right)$, respectively, form $\mathbf{C}$-bases for the unique subspaces of $X$ and $Y$ where the representation $\rho$ takes place.

Our conjecture is, therefore, that the two representation spaces are the same, i.e., $\rho$ appears only once in the representation of $\Gamma_{g}(1)$ in $X+Y$. This space is contained in the space, say $Z$, of all modular forms of weight $\frac{1}{2} g+1$ relative to $\Gamma_{g}(4,8)$ and the conjecture will follow if $\rho$ appears at most once, hence exactly once, in the representation of $\Gamma_{g}(1)$ in $\mathbf{Z}$.

In rounding off this article we shall explain an approach suggested to us by Mumford; as we understand it, the idea is as follows: in $D(M)$ and $P(N)$ we take the coefficients of $M$ and $N$ arbitrarily from $Z\left[\frac{1}{2}\right]$; if we can find a conjectural formula involving such $D(M)$ 's and $P(N)$ 's which is preserved under the action of $S p_{2 g}\left(Z\left[\frac{1}{2}\right]\right)$, then the problem will be reduced to the elliptic case in view of the fact that the $S p_{2 g}\left(Z\left[\frac{1}{2}\right]\right)$-orbit of $\sqrt{-1} 1_{g}$ is dense in $S_{g}$. We might mention that this is one of Poincarés ideas which we have recalled in Part 1. It is very likely that this idea, rediscovered by Mumford, will eventually settle the problem.

\section{REFERENCES}

1. A. A. Albert, A note on the Poincare theorem on impure Riemann matrices, Ann. of Math. (2) 36 (1935), 151-156.

2. P. Appell, Sur les fonctions périodiques de deux variables, J. Math. 7 (1891), 157-219.

3. W. L. Baily, Satake's compactification of $V_{n}$, Amer. J. Math. 80 (1958), 348-364.

4. H. Cartan, Fonctions automorphes, Séminaire E.N.S. (1957/58).

5. W.-L. Chow, On compact complex analytic varieties, Amer. J. Math. 71 (1949), 893-914.

6. J. Fay, On the Riemann-Jacobi formula, Göttingen Nachrichten (1979), 61-73.

7. E. Freitag, Siegelsche Modulfunktionen, Jahresber. Deutsch. Math.-Verein. 79 (1977), 79-86.

8. F. G. Frobenius, Über die Grundlagen der Theorie der Jacobischen Funktionen, Crelles J. 97 (1884), 16-48 \& 188-223; Werke II, 172-240.

9. __ Über die constanten Factoren der Thetareihen, Crelles J. 98 (1885), 244-263; Werke II, 241-260.

10. C. Hermite, Note sur la théorie des fonctions elliptiques (1862); Oeurvres II, 125-238.

11. J. Igusa, On the Picard varieties attached to algebraic varieties, Amer. J. Math. 74 (1952), 1-22.

12. __ On the graded ring of theta constants, Amer. J. Math. 86 (1964), 219-246.

13. __ On Jacobi's derivative formula and its generalizations, Amer. J. Math. 102 (1980), 409-446.

14. S. Kowalevski, Über die Reduktion einer bestimmten Klasse Abel'-scher Integrale $3^{\text {ten }}$ Ranges auf elliptische Integrale, Acta Math. 4 (1884), 393-414.

15. A. Krazer and W. Wirtinger, Abelsche Funktionen und allgemeine Thetafunktionen, Enzykl. Math. Wiss. II B 7 (1921), 604-873.

16. S. Lefschetz, On certain numerical invariants of algebraic varieties with application to abelian varieties, Trans. Amer. Math. Soc. 22 (1921), 327-482.

17. D. Mumford, On the equations defining abelian varieties. I-III, Invent. Math. 1 (1966), 287-354; 3 (1967), 75-135; 215-244.

18. $\_$Curves and their Jacobians, Univ. Mich. Press, 1975.

19. H. Poincaré, Sur les fonctions $\Theta$, Bull. Soc. Math. France 11 (1883), 129-134; Oeuvres IV, $302-306$.

20. H. Poincaré and E. Picard, Sur un théorème de Riemann relatif aux fonctions de $n$ variables indépendentes admettant $2 n$ systèmes de périodes, C. R. 97 (1883), 1284-1287; Oeuvres IV, 307-310. 
21. H. Poincaré, Sur la réduction des intégrales abéliennes, Bull. Soc. Math. France 12 (1884), 124-143; Oeuvres III, 333-351.

22. __ Sur les fonctions abéliennes, Amer. Math. 8 (1886), 289-342; Oeuvres IV, 318-378.

23. __ Remarques diverses sur les fonctions abéliennes, J. Math. 1 (1895), 219-314; Oeuvres IV, 384-468.

24. __ Sur les fonctions abéliennes, C. R. 124 (1897), 1407-1411; Oeuvres IV, 469-472.

25. _ Sur les propriétés du potentiel et sur les fonctions abéliennes, Acta Math. 22 (1898), 89-178; Oeuvres IV, 162-243.

26. __ Sur les fonctions abéliennes, Acta Math. 26 (1902), 43-98; Oeuvres IV, 473-526.

27. H. E. Rauch, Schottky implies Poincaré, Advances in the Theory of Riemann Surfaces, Ann. of Math. Studies, no. 66, Princeton Univ. Press, Princeton, N. J., 1971, pp. 355-364.

28. B. Riemann, Theorie der Abel'schen Functionen, Crelles J. 54 (1857), 115-155; Werke, pp. 88-142.

29. __ Werke; Nachträge by M. Noether and W. Wirtinger, 1902.

30. I. Satake, On the compactification of the Siegel space, J. Indian Math. Soc. 20 (1956), 259-281.

31. F. Schottky, Zur Theorie der abelschen Functionen von vier Variabeln, Crelles J. 102 (1888), 304-352.

32. J.-P. Serre, Faisceaux analytiques, Sém. Bourbaki 95 (1954).

33. C. L. Siegel, Uber die analytische Theorie der quadratischen Formen, Ann. of Math. (2) 36 (1935), 527-606; Werke I, 326-405.

34. Einführung in die Theorie der Modulfunktionen n-ten Grades, Math. Ann. 116 (1939), 617-657; Werke II, 97-137.

35. __ Meromorphe Funktionen auf kompakten analytischen Mannigfaltigkeiten, Göttingen Nachrichten (1955), 71-77; Werke III, 216-222.

36. K. Weierstrass, $\ddot{U}$ ber die allgemeinsten eindeutigen und $2 n$-fach periodischen Functionen von n Veränderlichen, Monatsbericht (1869), 854-857; Werke II, 45-48.

37. Allgemeine Untersuchungen über $2 n$-fach periodische Functionen von $n$ Veränderlichen; Werke III, 53-114.

38. A. Weil, Généralisation des fonctions abéliennes, J. Math. 17 (1938), 47-87.

39. __ Variétés abéliennes et courbes algébriques, Act. Sci. Ind. 1064 (1948).

40. __ Théorèmes fondamentaux de la théorie des fonctions thêta, Sém. Bourbaki 16 (1949).

41. W. Wirtinger, Zur Theorie der $2 n$-fach periodischen Funktionen, Monatsh. Math. 7 (1896), $1-25$.

Department of Mathematics, Johns Hopkins University, Baltimore, Maryland 21218 